\title{
THE EFFICACY OF GESTURAL CUEING IN DYSPHASIC WORD-RETRIEVAL RESPONSES
}

\author{
SAKINA S. DRUMMOND \\ University of Arkansas at Little Rock-University of Arkansas Medical Sciences \\ GARY J. RENTSCHLER \\ The University of Michigan, Ann Arbor
}

\begin{abstract}
The effectivenesss of visual-gestural cueing as compared with traditional auditory-verbal cueing was investigated using a time-series design. Eight dysphasic adults equally divided into a control and an experimental group were the subjects for this study. Results indicated no significant improvement in response times after an intensive 2 -wk treatment period. Similarly, no single cue was observed to be more effective than others in eliciting dysphasic word-retrieval responses. In contrast, there was a significant difference in the order in which different cues were presented. Findings indicated that regardless of cue type, the cue presented first was the most effective. The present discussion relates current findings to previous observations and reviews implications of the data for language rehabilitation in dysphasia.
\end{abstract}

\section{Introduction}

Word-finding difficulties persist across different types and severities of dysphasic impairment. A primary consideration in language rehabilitation addresses the treatment of word-finding difficultics. The use of specific input modalities and linguistic contexts has been studied in word-retrieval tasks. Pertinent studies investigating different cue types have identified specific types of cues as facilitators of word finding annong dysphasics. Barton, Maruszewski, and Urrea (1969) found sentence-completion cues effective facilitators in word-naming tasks. Love and Webb (1977) developed a cue effectiveness hierarchy on the basis of their examination of four different cue types in a picture-naming task. These investigators found word imitation the most effective cue, followed by initial syllable cues; sentence completion and printed word cues were equally effective, ranking third. Pease and Goodglass (1978) also used picture naming to investigate the effectiveness of six different cue types. Their results demonstrated that initial syllable cues proved more effective than sentence-completion cues, which ranked second. No significant difference in effectiveness was observed for the other four cue types (superordinate, function, location, and rhyme cues).

Address correspondence to Gary J. Rentschler, The University of Michigan, 1111 East Catherine Street, Ann Arbor, Michigan 48109.

Elsevier North Holland, Inc., 1981 
Each of these studies suggests that initial syllable and sentence-completion cues are more facilitatory than are other types of cueing. In addition, initial syllable cues were found more effective than sentence-completion cues (Love and Webb, 1977; Pease and Goodglass, 1978). Further investigation is needed to clarify the role of input-output modalities of cue presentation. Although initial syllable and sentence-completion cues represent phonologic and semantic components, respectively, they share a common modality of transmission; both are auditory - verbal in nature. Luria (1970) refers to auditory-verbal modality cucs as "intrasystemic." Intrasystemic functions process information in the same modality (input-out), whereas "intersystemic" processes utilize the integration of modalities. Therefore, in a cueing task in which the subject receives a spoken cue, e.g., sentence completion, for which a verbal response is required, the input-output process primarily uses the auditory-verbal modality. Intersystemic tasks may be exemplified by receiving a visual cue, such as a gesture, and requiring a verbal response. Interest in alternate transmission modalities has recently focused on nonverbal communicative systems. Duffy, Duffy, and Pearson (1975) investigated the relationship between nonverbal communicative symbol systems (pantomime recognition) and verbal abilities in dysphasia. Their findings characterized dysphasia as a reduction in overall functioning, effecting visual-gestural modality functions (signing, pantomiming, and gesturing), as well as auditory - verbal modality functions. Goodglass and Kaplan (1963) and Pickett (1972) impaired gesture and pantomine abilities among dysphasics. The Porch Index of Communicative Abilities (Porch, 1967) is also designed to be sensitive to visual-gestural disturbances. Notation of depressed performances on these subtests is not uncommon to the practicing clinician. Eagleson, Vaughn, and Knudson (1967) and Chen (1968) support the use of visual-gestural systems in overcoming verbal deficits in dysphasia.

The primary purpose of the present investigation was to examine the effectiveness of visual-gestural cueing in dysphasic naming responses as compared with traditional auditory-verbal cueing techniques. Two types of auditory-verbal cues and one visual-gestural cue were studied. These are the specific questions posed:

1. Is there a significant difference in the word-retrieval response times in dysphasic subjects receiving all three cue types as compared with those dysphasics receiving only auditory - verbal cues (initial syllable and sentence completion)?

2. Is there a significant difference among the three cueing techniques in aiding dysphasic word retrieval?

3. Does the order of presentation of different cue types make a significant difference in dysphasic word-retrieval responses? 


\section{Subjects}

Eight dysphasic adults participating in the Residential Aphasia Program at The University of Michigan, Communicative Disorders Clinics were the subjects in the study. Subjects ranged in age from $46 \mathrm{yr} 7 \mathrm{mo}$, to $59 \mathrm{yr}, 7 \mathrm{mo}$ (Table 1). The time elapsed from onset of dysphasia is also indicated in Table 1. Subjects were randomly divided into two groups, one group that would eventually receive gestural communication training (experimental group) and one that would not (control group).

\section{Stimuli}

The AMERIND Gestural Code (Skelly, Schinsky, Smith, and Fust, 1974; Skelly, 1979) was the visual-gestural system selected (AMER-American; INDIndian). The AMERIND code was chosen because it is standardized and reliable and because it would facilitate instruction. The AMERIND signs represent a salient feature of an item in terms of its function, shape, or relative location.

Thirty common nouns, controlled for word frequency and picturability were selected as stimuli for the study. Twenty of the 30 words were items found in the AMERIND dictionary. All 30 were pictured in the Martha Sarno Taylor Aphasia Therapy Kit (Taylor and Marks, 1959), which was used as stimulus material for testing.

A pretest was administered to ensure that subjects had demonstrable wordfinding difficulty; it also served as a pretreatment measure of response time. A picture-naming task composed of 10 of 20 AMERIND nouns and 10 other nouns

TABLE 1

Subject Ages and Time Elapsed Since Onset

\begin{tabular}{|c|c|c|}
\hline Subjects & Age & $\begin{array}{c}\text { Time } \\
\text { Postonset }\end{array}$ \\
\hline \multicolumn{3}{|l|}{ Experimental group } \\
\hline P.M. & $56 \mathrm{yr}, 2 \mathrm{mo}$ & $16 \mathrm{mo}$ \\
\hline K.L. & $46 \quad 10$ & 20 \\
\hline D.J. & 59 & 10 \\
\hline P.D. & 48 & 15 \\
\hline \multicolumn{3}{|l|}{ Control group } \\
\hline M.K. & $50 \mathrm{yr}, 6 \mathrm{mo}$ & $24 \mathrm{mo}$ \\
\hline W.L. & $56 \quad 3$ & 15 \\
\hline B.N. & 46 & 9 \\
\hline D.D. & 46 & 23 \\
\hline
\end{tabular}


was used during the pretesting phase. When shown a stimulus picture, subjects were asked to name the picture as quickly as possible. The carrier phrase, "What is this?," was asked simultaneously as the picture was shown. An error rate of $10 \%$ was established as the minimum level to be considered indicative of word-finding difficulty. All subjects met the criterion for inclusion in the study.

The experimental group received two 20-min training sessions during which they were instructed in the use of the AMERIND signs for the 20 stimulus nouns selected. All subjects in this group demonstrated comprehension of the signs and were able to use them accurately to represent each noun $95 \%$ of the time prior to the treatment sessions. The control group received no pretreatment instruction.

All subjects received treatment sessions that varied in length from 15 to $30 \mathrm{~min}$ daily for a period of $2 \mathrm{wk}$. The sessions were designed to drill word-retrieval skills, using cueing when necessary. The control group received traditional initial-syllable and sentence-completion cues, whereas the experimental group received AMERIND cues in addition to the aforementioned traditional cue types. A standardized cueing protocol was established to ensure that each subject received identical cues. These protocols are presented in the Appendix. Description of cue types appears in Table 2.

The presentation order of cue types was randomized for each treatment session. For example, if a subject in the experimental group was unable to name a picture stimulus, the designated sentence completion cue was given first; if the subject was unable to retrieve the word, the AMERIND cue was given; and if the subject was still unable to name the item, the initial syllable cue was presented. During the next session, the presentation order of cue types was changed. The task was discontinued when the subject successfully retrieved the desired word or after each cue type had been presented once.

TABLE 2

Description of Cues

\begin{tabular}{|c|c|c|}
\hline Type of Cue & Definition & $\begin{array}{c}\text { Example } \\
\text { (comb) }\end{array}$ \\
\hline \multicolumn{3}{|l|}{ Auditory-verbal } \\
\hline Initial syllable & $\begin{array}{l}\text { The initial consonant and vowel } \\
\text { of the target word. }\end{array}$ & $" / \mathrm{kol} "$, \\
\hline Sentence completion & $\begin{array}{l}\text { An open-ended sentence } \\
\text { requiring the target word for } \\
\text { completion. }\end{array}$ & "You fix your hair with a \\
\hline \multicolumn{3}{|l|}{ Visual-gestural } \\
\hline AMERIND & A manual gesture or sign. & $\begin{array}{l}\text { Left hand elevated to left side of } \\
\text { head, fingers spread, palm down. } \\
\text { Then moved downward, toward } \\
\text { the ear, three times. }\end{array}$ \\
\hline
\end{tabular}


Following the 2-wk treatment period, post-testing was administered to each dysphasic subject. The post-test was also composed of 10 of the 20 AMERIND nouns plus 10 non-AMERIND nouns included to measure possible generalization effects of treatment. Both the pre- and post-tests were tape recorded.

The tapes were played back to permit measurement of response time. Response times were measured independently by two examiners, each of whom had a stopwatch. The time from the end of the carrier phrase to onset of vocalization of the correct answer was timed to the nearest tenth of a second. Examiner agreement within $0.2 \mathrm{sec}$ was required for each response. The examiners' measurements were averaged for each response. The pre- and post-treatment response times (the average of the two examiners) are presented in Table 3.

\section{Data Analysis}

A series of 21 paired $t$-tests were administered to analyze the results. The difference between pre- and post-treatment response times and the frequency of correct responses during the treatment period served as dependent variables. The independent variables included the three cue types (sentence completion, initial syllable, and AMERIND), as well as the presentation order of cues, and the dysphasic subjects.

\section{Results and Discussion}

\section{Response Times}

Whereas the present data showed an improvement in the pre- and posttreatment response times for all eight dysphasic subjects, these changes were not statistically significant $(\bar{X}=3.66 \mathrm{sec} ; t=4.93 ; p<0.2)$. Contrasting the

TABLE 3

Subject Response Times (in seconds)

\begin{tabular}{lrc}
\hline \multicolumn{1}{c}{ Subjects } & Pretreatment Times & Postreatment Times \\
\hline Experimental group & & \\
P.M. & 2.35 & 2.82 \\
K.L. & 1.26 & 0.33 \\
D.J. & 54.43 & 33.53 \\
P.D. & 0.73 & 0.51 \\
Control group & & \\
M.K. & 14.69 & 9.09 \\
W.L. & 0.60 & 0.32 \\
B.N. & 1.71 & 0.51 \\
D.D. & 0.91 & 0.35 \\
\hline
\end{tabular}


treatment groups, the experimental group demonstrated greater improvement in response times $(\bar{X}=5.39 \mathrm{sec})$ than did the control group $(\bar{X}=1.91 \mathrm{sec})$; however, their difference failed to reach statistical significance $(t=3.25$; $p<0.2$ ) (Fig. 1).

It does not necessarily follow from these findings that the inclusion of a third cue fails to enhance dysphasic word-retrieval responses. The present investigation hypothesized that improvement in response time would be a sensitive index for assessing word-retrieval ability. This contention could have been inaccurate, as the results indicated that dysphasic response times did not improve significantly with treatment. These findings might question the validity of using response time as a criterion for making observations of behavioral changes.

An argument for this assertion relates to the influence of individual differences and styles inherent in the word-retrieval process. Each speaker develops and uses a particular verbal style that becomes imprinted in verbal behavior. Verbal style includes such parameters as response latency, pausing, retrieval strategy, and hesitations, generally referred to as paralinguistic behaviors. Whereas dysphasic speakers manifest obvious linguistic impairments, change or impairment of

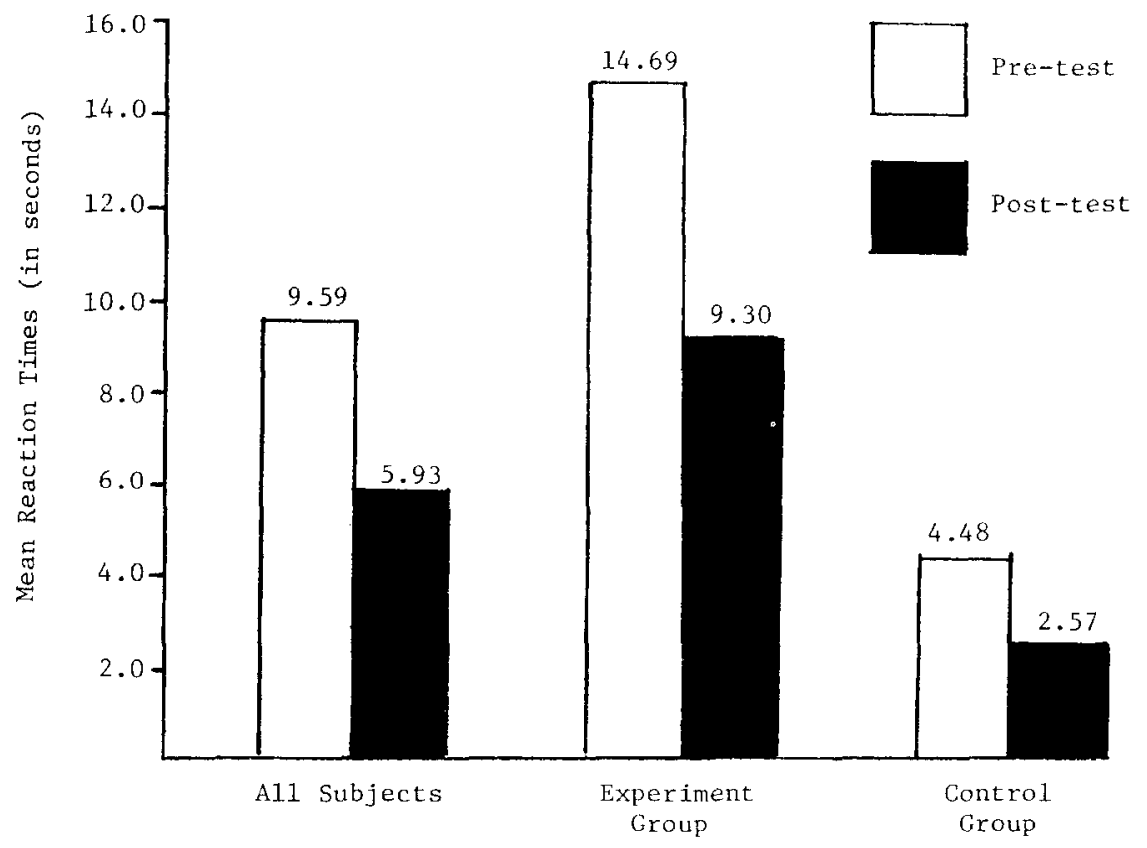

Fig. 1. Improvement in response times; all subjects, $3.66 \mathrm{sec}$; experiment group, $5.39 \mathrm{sec}$; control group, $1.91 \mathrm{sec}$. 
paralinguistic behaviors cannot necessarily be inferred. For example, someone speaking with both great deliberation and a long response latency prior to dysphasia might be more inclined to continue to use a similar verbal style postonset. We would therefore be hasty to conclude that this person is likely to shorten response time within so relatively brief a treatment course.

Second, speed as a measure of the word-retreival process fails to describe the variability encountered in each retrieval attempt. Although most subjects experienced some successful retrieval attempts within a normal response time range, there is no indication of the cause of the increased response times in other instances. It is not known whether the additional time was required for searching, formulation, self-correction, recognition, or other aspects of impaired naming as discussed by Mills, Knox, Joula, and Salmon (1979). At this point in our understanding of word retrieval in dysphasia, we must acknowledge the complexity of the process, which is masked by its simplicity to the nondysphasic communicator.

\section{Cue Modalities}

Figure 2 graphically represents the effect of cue type in eliciting correct responses. Within the experimental group, initial syllable cues produced a greater frequency of accurate responses than did either sentence completion $(t=$

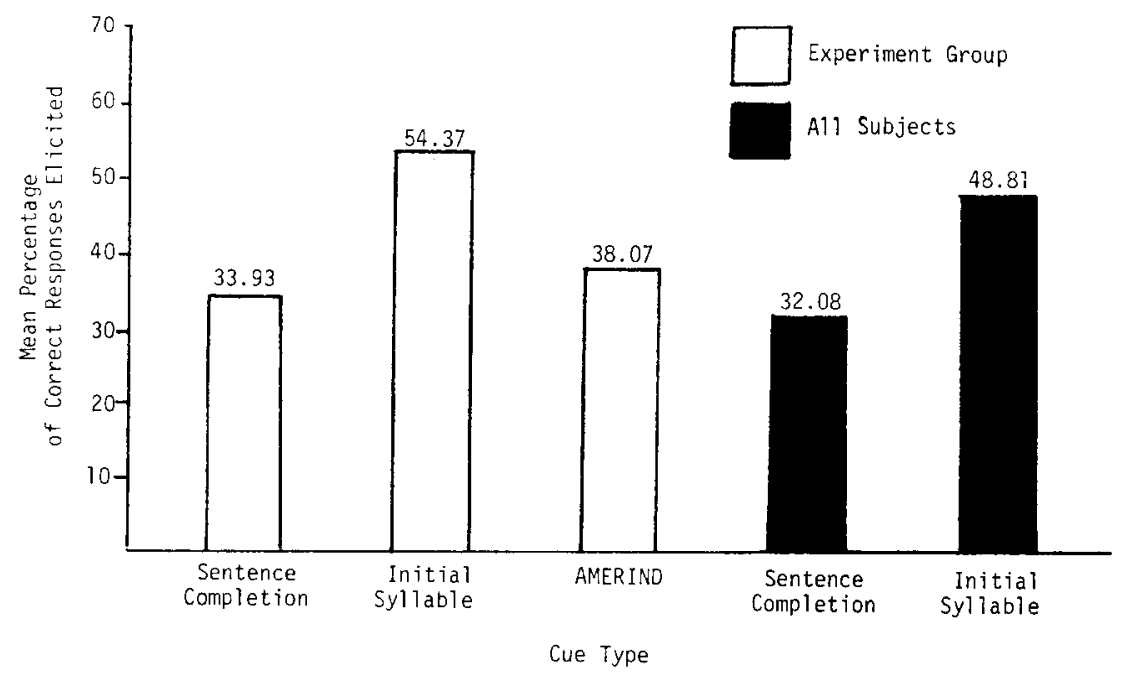

Fig. 2. The effect of cue type in eliciting responses. 
$2.33 ; p<0.2)$ or AMERIND cues $(t=1.61 ; p<0.4)$. However, this difference between the cue types was not statistically significant.

The auditory-verbal cues, initial syllable and sentence completion, were compared across all eight subjects, as these cueing techniques were used with both the experimental and control groups. Again, whereas initial syllable cues produced a greater frequency of accurate responses than did sentence completion cues, the difference failed to attain statistical significance $(t=1.04 ; p<0.4)$. Intuitively, the initial syllable cue would seem to be somewhat stronger, as it supplies part of the correct response for the subject. The results seemed to confirm this supposition partially, but apparently the sentence-completion format uses the structure of language to facilitate word retrieval with nearly equal success.

Other findings tended to indicate that supplementing a traditional auditory-verbal modality with a visual-gestural modality cue does not significantly facilitate word-retrieval response. In other words, AMERIND signs as a cueing technique do not enhance the word-retrieval ability of dysphasic subjects. This specific observation has implications for Luria's (1970) intrasystemic and intersystemic reorganization hypothesis. The present investigation consistently sought to elicit verbal modality response, varying the auditory-verbal and visual-gestural cues. The data suggest that dysphasics as a group do not show a demonstrable difference using either intrasystemic or intersystemic forms of cueing. One might conclude that intrasystemic, i.e., visual-gestural, reorganization could have some perceptual value during the decoding (input) process, but not necessarily for encoding.

The lack of a significant difference between cue types found in this study was not consistent with the findings reported by Love and Webb (1977) and Pease and Goodglass (1978). In each of these earlier studies initial syllable cues were found to be significantly more effective than were sentence completion cues. One explanation for these conflicting findings might be related to differences in experimental designs. The current investigation compared the two cueing techniques on the basis of dysphasics' word-retrieval responses during a 2-wk treatment period, whereas results from previous studies relied on dysphasic word-retrieval responses in a single test-oriented situation.

Second, the selective facilitatory effect of initial syllable cues noted in previous literature could be related to specific types of dysphasia. Luria (1970) reported that motor (Broca's) aphasics tend to present a greater difficulty with the articulatory components of the target word and therefore benefit more from initial syllable cues. Love and Webb (1977) confirmed these observations in their study, which included only Broca's asphasics. The present investigation did not categorize the dysphasic subjects because none of the selected subjects fully characterized a specific diagnostic category. 


\section{Cue Order}

When the effect of cue order was analyzed, the results indicated that the first cue presented, regardless of cue type, yielded a greater success rate in eliciting accurate responses. Figure 3 represents the effect of cue presentation order for all eight dysphasic subjects. Here the frequency of correct responses elicited on presentation of the first cue was significantly greater than those elicited following the second cue $(t=36.18 ; d f=7 ; p<0.001)$.

The effect of cue presentation order was also compared in the experimental group, which also received a third cue. Again, the cue presented first produced a significantly greater frequency of accurate responses than did either the second or third cue $(t=4.19 ; p<0.05$ and $t=4.72 ; p<0.02)$. No significant difference in the frequency of correct responses was found between the second and third cues $(t=1.94 ; p<0.2)$.

These findings indicate that irrespective of cue type, the cue presented first to the dysphasic subject is most likely to aid word retrieval. Whereas provision of multiple cues occasionally did facilitate retrieval, the general effectiveness of multiple cueing was limited.

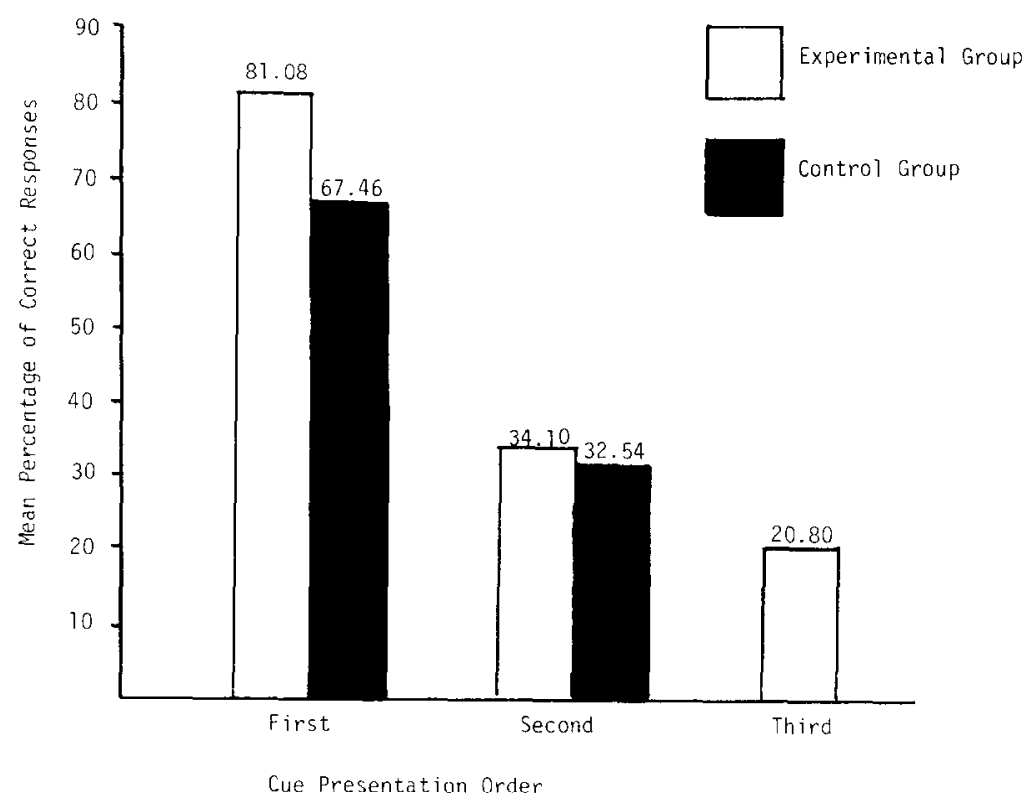

Fig. 3. The effect of cue order in eliciting responses. 
The present findings have significant clinical implications. The facilitatory effects of sclective cues as identificd in other isolated test-oriented situations (e.g., Love and Webb, 1977; Pease and Goodglass, 1978) conflict with current observations made in day-to-day treatment situations. Dysphasics' retrieval responses obtained from the 2 -wk treatment period clearly indicate that if the dysphasic is to retrieve a target word successfully with the assistance of a cue, it will most likely be with the first cue presented, regardless of cue type. The relative effectiveness of subsequent cues was observed to be minimal.

\section{Severity of Dysphasia}

The responses obtained from the initial syllable and sentence-completion conditions were examined in terms of the severity of language impairment (Figure 4). The eight subjects were grouped by severity level, as determined by their performance on the Boston Diagnostic Aphasia Examination (Goodglass

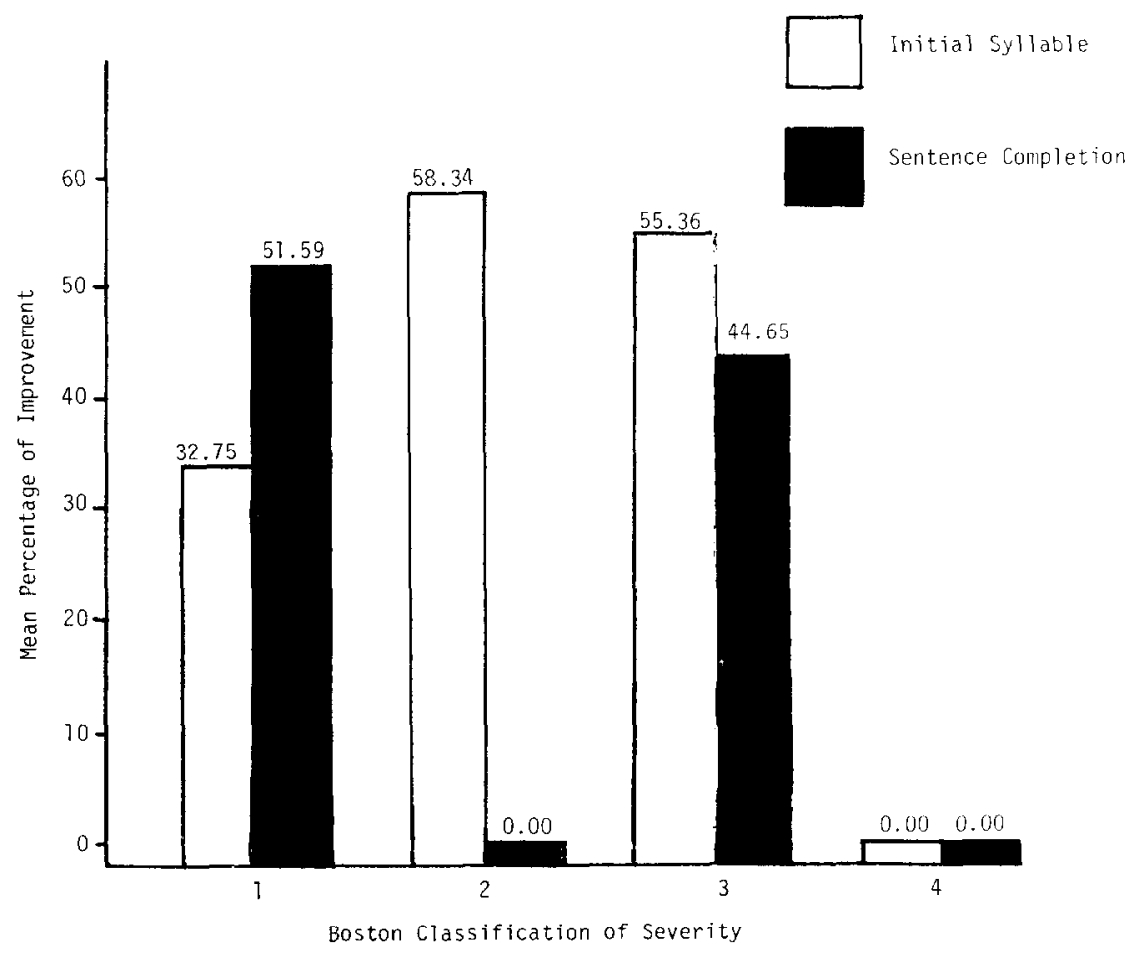

Fig. 4. Cueing efficacy by severity of language impairment. 
and Kaplan, 1972). Those subjects assigned to severity level 1 had significantly greater success using sentence completion cues than with initial cues $(t=14.84$; $\mathrm{df}=1 ; p<0.05$ ). By contrast, subjects assigned to level 2 had significantly greater success with initial syllable cues than with sentence-completion cues $(t=$ 9.87; $d f=1 ; p<0.1$. Subjects assigned to levels 3 and 4 did not show a significant preference for either of the two cues.

This finding suggests that whereas the facilitatory effects of cue type remain nonsignificant in mildly impaired dysphasics, a definite relationship exists between the facilitatory effects of specific cue types and the more severely impaired dysphasics. Furthermore, these findings do not support the traditional contention that dyspraxic subjects are greatly facilitated by initial syllable cues (Love and Webb, 1977; Pease and Goodglass, 1978), because the subjects assigned to level 1 were identified with dyspraxic symptoms. The present data, however, are derived from a very small sample size (two subjects at each severity level) and therefore warrant further investigation.

In summary, the present investigation indicates that gesturing as an intersystemic form of reorganization does not make a significant difference in facilitating dysphasic word-retrieval responses. Such nonsignificant findings lend credence to previous observations that gestural communication is an integral component of all propositional speech. Thus our results support the contention that dysphasia affects all language modalities. Although it was indicated that dysphasics as a group fail to benefit from one specific cue type, some selective facilitatory effect of cue type may exist for more severely language-impaired subjects. Finally, and most importantly, the present data clearly indicate that irrespective of the cue type, the cue presented first was most significant in aiding word retrieval.

\section{Appendix: Cueing Protocols for Sentence Completion Cues}

1. You live in a (house).

2. You drive a (car).

3. You call people on a ___ (telephone).

4. You sit on a (chair).

5. You drink tea from a (cup).

6. You unlock the door with a (key).

7. To help them read, people wear

8. You fix your hair with a (glasses).

9. You read a (comb).

10. You buy things with (book).

11. A boy grows up to become a

12. A girl grows up to become a

13. You clean your teeth with a

14. You pound nails with a

a

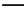
(money). (man). (woman). (toothbrush). (hammer). 
15. You pick meat up with a (fork).

16. You ring a (bell).

17. You cut meat with a (knife).

18. You take pictures with a (camera).

19. You wash your hands with soap and (water).

20. On your finger you wear a (ring).

\section{References}

Barton, M. I., Maruszewski, M., and Urrea, D. (1969). Variation of stimulus context and its effect on word finding ability of aphasics. Cortex 5:351-365.

Chen, L. Y. (1968). Talking hand of aphasia stroke patients. Geriatrics 23:145-148.

Duffy, R., Duffy, J., and Pearson, K. (1975). Pantomime recognition in aphasics. J. Speech Hear. Res. 18:115-129.

Eagleson, R. M., Vaughn, G., and Knudson, A. (1967). Hand signals for dysphasia. Arch Phys. Med. Rehab. 48:410-414.

Goodglass, H., and Kaplan, E. (1963). Disturbance of gesture and pantomine in aphasia. Brain 86:703-720.

Goodglass, H., and Kaplan, E. (1972), Boston Diagnostic Aphasia Examination. Philadelphia: Lea and Febiger.

Love, R., and Webb, W. (1977). The efficacy of cueing techniques in Broca's aphasia. I. Speech Hearing Dis. 42:170-178.

Luria, A. (1970). Traumatic Aphasia: Its Syndromes, Psychology and Treatment. The Hague: Mouton.

Mills, R. H., Knox, A. W., Juola, J. F., and Salmon, S. J. (1979). Cognitive loci of impairments in picture naming by aphasic subjects. J. Speech Hearing Res. 22:73-87.

Pease, D., and Goodglass, H. (1978). The effects of cueing on picture naming in aphasia. Cortex 14:178-189.

Pickett, L. (1972). An assessment of gestural and pantomimic deficit in aphasic patients. M.A. Thesis. University of New Mexico.

Porch, B. (1967). Porch Index of Communicative Ability. Palo Alto, CA: Consulting Psychologists Press.

Skelly, M., Schinsky, L., Smith, R., and Fust, R. (1974). American Indian sign (Amerind) as a facilitator of verbalization for the oral verbal apraxic. J. Speech Hear. Dis. 39:445-456.

Skelly, M. (1979). Amer-Ind Gestural Code Based on Universal American Indian Hand Talk. New York: Elsevier.

Taylor, M., and Marks, M. (1959). Aphasia Rehabilitation Manual and Therapy Kit. New York: McGraw-Hill. 